\title{
A Note on Solving a Fully Intuitionistic Fuzzy Linear Programming Problem based on Sign Distance
}

\author{
S. K. Bharati \\ Department of Mathematics, \\ Faculty of Science \\ Banaras Hindu University, \\ Varanasi-221005, India
}

\author{
S. R. Singh \\ Department of Mathematics, \\ Faculty of Science \\ Banaras Hindu University, \\ Varanasi-221005, India
}

\begin{abstract}
The paper presents a new method to find the optimal solution of a fully intuitionistic fuzzy linear programming (FIFLP) problem. It uses the sign distance between intuitionistic fuzzy numbers for their comparison. The proposed methods have been applied for solving a FIFLP problem with equality constraints. The proposed method is convenient for implementation to solution of FIFLP problems arising in real life situations.
\end{abstract}

\section{General Terms}

Linear programming, distance function.

\section{Keywords}

Intuitionistic fuzzy sets, Triangular intuitionistic fuzzy numbers, Sign distance between intuitionistic fuzzy numbers, Fully intuitionistic fuzzy linear programming problem.

\section{INTRODUCTION}

Bellman and Zadeh [3] first introduced concept of the fuzzy set given by Zadeh [8] to consider solution of decision making problems with imprecision in parameters. Later Zimmermann [28] considered these imprecise parameters as fuzzy numbers and formulated as fuzzy linear programming problem. Since fuzzy linear programming problems consist of fuzzy numbers and fuzzy inequalities, the comparison between these of fuzzy parameters arising in the problem are not straightforward. The comparison of fuzzy parameters needed the ranking methods for their quantitative comparison. Several methods have been developed for ranking of fuzzy numbers and are available in literature given by Cheng [9], Yao and $\mathrm{Wu}$ [10], Yao and Lin [27], Tran and Duckstein [11], Chu, Ta-Chun, Tsao, C. Tsen [12], Thus Mitchell [7], Grzegorzewski [13], Abbasbandy and Asadi [14], Shureshjani and Darehmiraki [15]. Several methods were also developed to find solution of fuzzy linear programming problems using ranking methods. Further, in modeling the decision making problems with uncertainty vagueness and imprecision, it was found that their representation by fuzzy numbers need some alternative methods. For general class of decision making problem with membership, non-membership and hesitation function. Atanassov [1] considered the problem and generalised the fuzzy set as intuitionistic fuzzy set to deal with imprecision in a better way. Intuitionistic fuzzy set also takes care of degree of imprecision along with degree of belonging and non belonging. It was Angelov [2] who extended the concept of fuzzy optimization to intuitionistic fuzzy optimization. Further, since these problems of intuitionistic fuzzy optimization contain the imprecise parameters as intuitionistic fuzzy numbers, their quantitative comparison need the ranking methods. There are several ranking methods for intuitionistic fuzzy numbers and are available in literature such as Szmidt and Kacprzyk [16], Wang and Xin [17], Nayagam et at [18], [20], Li [6], Li Nan, Zhang [19], Wei and Tang [21], Nehi [22], De and Das [23]. A distance based ranking of intuitionistic fuzzy numbers was developed by Guha and Chakrborty [25], Esmailzadeh [26], Zhang and $\mathrm{Xu}$ [24]. Further, in many situations many real life problems comprise of intuitionistic fuzzy numbers as well as inequalities and thus such problems are fully intuitionistic fuzzy in nature and need separate attention for its solution. Recently some authors have worked on intuitionistic fuzzy linear programming problems such as Dubey and Aparna Mehra [4], Bharati and Singh [5]. Thus motivated with above studies in this paper we considered a method of ranking intuitionistic fuzzy numbers based on its distance from the intuitionistic fuzzy origin. The method is simple and need to compute membership function for its ranking. We have also used the proposed ranking method to solve a intuitionistic fuzzy linear programming problem for its applicability.

The remainder of this paper is organized as follows. In section 2 , we firstly stated preliminary definitions and some basic notions related to intuitionistic fuzzy numbers. In section 3, we have defined fully intuitionistic fuzzy linear programming which is not already occurred in literature. In section 4, we have found distance of an intuitionistic fuzzy number from intuitionistic fuzzy origin. In section 5 , we have stated and proved some theorems/results. Section 6, presents new algorithm based on signed distance. In section 7, we illustrated the algorithm. In section 8, we have given conclusion.

\section{PRELIMINARIES}

\subsection{Intuitionistic fuzzy sets}

Let a non fuzzy set $X$ be fixed. An intuitionistic fuzzy set (IFS) $\mathrm{A}$ in $\mathrm{X}$ is defined as object of the following form $\tilde{A}^{I}=\left\{\left(x, \mu_{\tilde{A}^{I}}(x), v_{\tilde{A}^{I}}(x)\right): x \in X\right\}$, where $\quad \mu_{\tilde{A}^{I}}: X \rightarrow[0,1]$ and $v_{\tilde{A}^{I}}: X \rightarrow[0,1]$ define the degree of membership and the degree of non membership of the element $x \in X$, respectively and for every $x \in X, 0 \leq \mu_{\tilde{A}^{I}}(x)+v_{\tilde{A}^{I}}(x) \leq 1$.

The value of $\pi_{\tilde{A}^{I}}(x)=1-\mu_{\tilde{A}^{I}}(x)-v_{\tilde{A}^{I}}(x)$, is called the degree of non-determinacy (or uncertainty) of the element $\mathrm{x}$ $\in \mathrm{X}$ to the intuitionistic fuzzy set A.

When $\pi_{\tilde{A}^{I}}(x)=0$, then an intuitionistic fuzzy set becomes fuzzy set.

$$
\left\{\left(x, \mu_{\tilde{A}^{I}}(x), 1-\mu_{\tilde{A}^{I}}(x)\right): x \in X\right\} .
$$




\subsection{Intuitionistic fuzzy number}

An intuitionistic fuzzy set $\tilde{A}^{I}=\left\{\left(x, \mu_{\tilde{A}^{I}}(x), v_{\tilde{A}^{I}}(x)\right): x \in \mathbb{R}\right\}$ of the real number is called intuitionistic fuzzy number if

(i) There exit a real numbers $x_{0} \in \mathbb{R}$ such that $\mu_{\tilde{A}^{I}}\left(x_{0}\right)=1$, and $v_{\tilde{A}^{I}}\left(x_{0}\right)=0$,

(ii) Membership $\mu_{\tilde{A}^{I}}$ of $\tilde{A}^{I}$ is fuzzy convex and non-membership $v_{\tilde{A}^{I}}$ of $A$ is fuzzy concave.

(iii) $\quad \mu_{\tilde{A}^{I}}$ is upper semi-continuous and $v_{\tilde{A}^{I}}$ is lower semi-continuous.

(iv) Support $\quad\left(\tilde{A}^{I}\right)=\overline{\left(\left\{x \in \mathbb{R}: v_{\tilde{A}^{I}}(x)<1\right\}\right)}$ is bounded.

\subsection{Triangular intuitionistic fuzzy number}

Triangular intuitionistic fuzzy number is denoted by $\tilde{A}^{I}=$ $\left\{\left(a_{1}, a_{2}, a_{3}\right),\left(b_{1}, b_{2}, b_{3}\right)\right\}$, where $a_{1}, a_{2}, a_{3}, b_{1}, b_{2}, b_{3} \in \mathbb{R}$ suchthat $b_{1} \leq a_{1} \leq a_{2} \leq b_{2} \leq a_{3} \leq b_{3}$ is an intuitionistic fuzzy numbers having membership and non-membership functions is of form

$\mu_{\tilde{A}^{I}}(x)=\left\{\begin{array}{cc}1, & x=a_{2} \\ 0, & x \leq a_{1}, x \geq a_{3} \\ f_{1}(x), & a_{1}<x<a_{2}, \\ f_{2}(x), & a_{2}<x<a_{3}\end{array}\right.$ and

$v_{\tilde{A}^{I}}(x)=\left\{\begin{array}{lr}1, & x=a_{2} \\ 0, & x \leq a_{1}, x \geq a_{3} \\ g_{1}(x), & b_{1}<x<b_{2}, \\ g_{2}(x), & b_{2}<x<b_{3}\end{array}\right.$

Where

$f_{1}:\left(a_{1}, a_{2}\right) \rightarrow[0,1], f_{2}:\left(a_{2}, a_{3}\right) \rightarrow[0,1], g_{1}:\left(b_{1}, b_{2}\right) \rightarrow$ $[0,1], g_{2}:\left(b_{2}, b_{3}\right) \rightarrow[0,1]$.

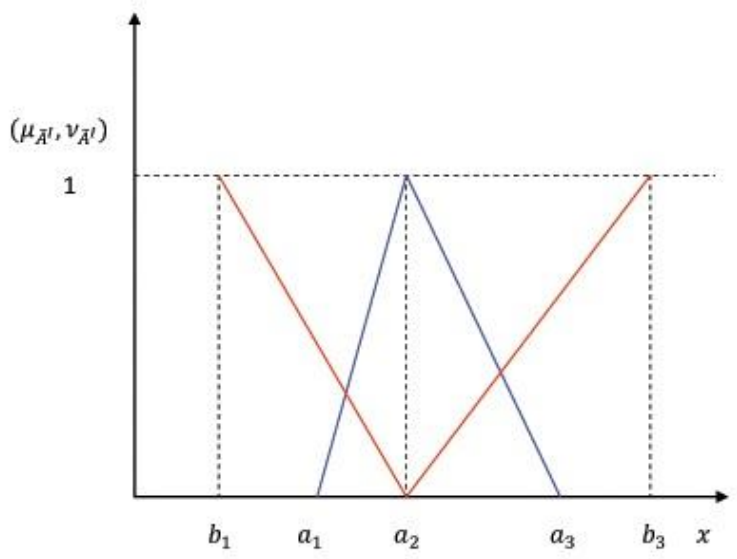

Figure 1: Triangular intuitionistic fuzzy number.

\subsection{Positive triangular intuitionistic fuzzy} number

Triangular intituitonistic fuzzy numbers $\tilde{A}^{I}=\left\{\left(a_{1}\right.\right.$, $\left.\left.a_{2}, a_{3}\right),\left(b_{1}, a_{2}, b_{3}\right)\right\}$ is said to be positive triangular intuitionitsic fuzzy number if $b_{1}>0$.

\subsection{Some properties of triangular intuitionistic fuzzy numbers}

Property 1.

Let $\quad \tilde{A}^{I}=\left\{\left(a_{1}, a_{2}, a_{3}\right),\left(b_{1}, a_{2}, b_{3}\right)\right\}$

and $\tilde{C}^{I}=\left\{\left(c_{1}, c_{2}, c_{3}\right),\left(d_{1}, c_{2}, d_{3}\right)\right\}$ be two triangular intuitionistic fuzzy numbers. Then addition of $\tilde{A}^{I}$ and $\tilde{B}^{I}$ is also a triangular intuitionistic fuzzy number and is defined as $\tilde{A}^{I} \oplus \tilde{C}^{I}=\left\{\left(a_{1}+c_{1}, a_{2}+c_{2}, a_{3}+c_{3}\right),\left(b_{1}+d, a_{2}+\right.\right.$ $\left.\left.c_{2}, b_{3}+d_{3}\right)\right\}$.

\section{Property 2.}

Let $\tilde{A}^{I}=\left\{\left(a_{1}, a_{2}, a_{3}\right),\left(b_{1}, a_{2}, b_{3}\right)\right\}$ be an intuitionistic fuzzy number and $k \in \mathbb{R}$, then scalar multiplication is also an intuitionistic fuzzy number and is given by

$k \tilde{A}^{I}=\left\{\begin{array}{l}\left\{\left(k a_{1}, k a_{2}, k a_{3}\right),\left(k b_{1}, k a_{2}, k b_{3}\right)\right\}, k>0 \\ \left\{\left(k a_{3}, k a_{2}, k a_{1}\right),\left(k b_{3}, k a_{2}, k b_{1}\right)\right\} k<0\end{array}\right.$.

Property 3.

Two intuitionistic fuzzy numbers $\tilde{A}^{I}=\left\{\left(a_{1}, a_{2}, a_{3}\right),\left(b_{1}, a_{2}, b_{3}\right)\right\} \quad$ and $\tilde{C}^{I}=\left\{\left(c_{1}, c_{2}, c_{3}\right),\left(d_{1}, c_{2}, d_{3}\right)\right\}$ are said to be equal iff $a_{1}=c_{1}, a_{2}=c_{2}, a_{3}=c_{3}, b_{1}=d_{1}, a_{2}=c_{2}, b_{3}=d_{3}$.

\subsection{Symmetrical intuitionistic fuzzy} number

Symmetrical intuitionistic fuzzy number is a intuitionistic fuzzy number whose membership function $\left(\mu_{\tilde{A}^{I}}\right)$ and nonmembership function $\left(v_{\tilde{A}^{I}}\right)$ are given by

$$
\mu_{\tilde{A}^{I}}(x)=\left\{\begin{array}{cc}
\frac{x-a_{1}}{a_{2}-a_{1}}, & a_{1}<x<a_{2} \\
1, & x=a_{2} \\
0, \quad x> & a_{3}, x<a_{1} \\
\frac{a_{3}-x}{a_{3}-a_{1}}, & a_{2}<x<a_{3}
\end{array},\right.
$$

and

$$
v_{\tilde{A}^{I}}(x)=\left\{\begin{array}{cc}
\frac{x-b_{2}}{b_{3}-b_{2}}, & b_{2}<x<b_{3} \\
0, & x=b_{2} \\
1, & x>b_{3}, x<b_{1} \\
\frac{b_{2}-x}{b_{2}-b_{1}}, & b_{1}<x<b_{2}
\end{array}\right.
$$

Where $b_{1} \leq a_{1} \leq a_{2} \leq b_{2} \leq b_{3}$ and $a_{2}-a_{1}=a_{3}$ $a_{2}, b_{2}-b_{1}=b_{3}-b_{2}, \quad a_{1}, a_{2}, a_{3}, b_{1}, b_{2}, b_{3} \in \mathbb{R}$. 


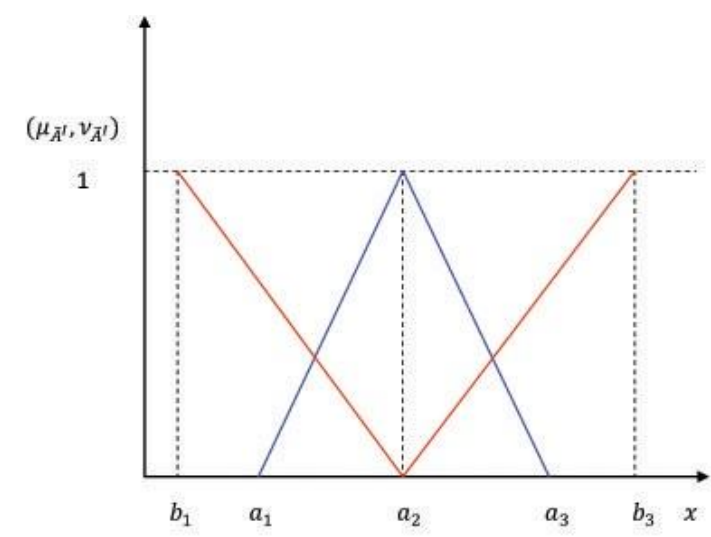

Figure 2: Symmetric triangular intuitionistic fuzzy number.

\section{FULLY INTUITIONISTIC FUZZY}

\section{LINEAR PROGRAMMING PROBLEMS}

A linear programming problem in which all the parameters are intuitionistic fuzzy numbers is called fully intuitionistic fuzzy linear programming. In mathematical formulation of a fully intuitionistic fuzzy linear Programming be considered as:

The new proposed method finds the optimal solution of the IFLP problem:

$$
\left.\begin{array}{rl}
\text { Maximize (or Minimize) } Z & =\tilde{c}^{I F} \otimes \tilde{x}^{I F} \\
\text { S.t. } \quad \tilde{a}^{I F} \otimes \tilde{x}^{I F} & =\tilde{b}^{I F} \\
\tilde{x}^{I F} & \geq \tilde{0}^{I F} .
\end{array}\right\}
$$

In problem (1), $\tilde{c}^{I F}, \tilde{a}^{I F}, \tilde{b}^{I F}$, are triangular intuitionistic fuzzy numbers.

\section{SIGN DISTANCE BETWEEN TWO TRIANGULAR INTUITIONISTIC \\ FUZZY NUMBERS}

\subsection{Intuitionistic fuzzy origin}

When $a_{1}=a_{2}=a_{3}=b_{1}=b_{2}=b_{3} \in \mathbb{R}$, Then $\tilde{A}^{I}=$ $\left\{\left(a_{1}, a_{2}, a_{3}\right),\left(b_{1}, b_{2}, b_{3}\right)\right\}$ is called intuitionistic fuzzy origin. In our paper, we have denoted intuitonistic fuzzy origin by $\tilde{0}^{I}$. And its parametric form is $\bar{u}(\alpha)=\underline{u}(\alpha)=\bar{v}(\alpha)=\underline{u}(\alpha)=0$.

4.2. Sign distance of triangular intuitionistic fuzzy number from intuitionistic fuzzy origin:

Let $\quad \tilde{A}^{I}=\left\{\left(a_{1}, a_{2}, a_{3}\right),\left(b_{1}, a_{2}, b_{3}\right)\right\} \quad$ be triangular intuitionistic fuzzy number. Then sign distance of $\tilde{A}^{I}$ can be calculated as

$$
\begin{aligned}
D^{S}\left(\tilde{A}^{I}, \tilde{0}^{I}\right)=\frac{1}{4}\left[\int_{0}^{1}\right. & \left(\tilde{A}_{L}^{+}(\alpha)\right) d \alpha \\
& +\int_{0}^{1}\left(\tilde{A}_{U}^{I^{+}}(\alpha)\right) d \alpha \\
& \left.+\int_{0}^{1}\left(\tilde{A}_{L}^{I^{-}}(\alpha)\right) d \alpha+\int_{0}^{1}\left(\tilde{A}_{U}^{I_{U}^{-}}(\alpha)\right) d \alpha\right]
\end{aligned}
$$

Where, $\quad \tilde{A}_{L}^{I^{+}}(\alpha), \tilde{A}_{U}^{I^{+}}(\alpha), \tilde{A}_{L}^{I^{-}}(\alpha), \tilde{A}_{U}^{I_{U}^{-}}(\alpha)$ is parametric triangular intuitionistic fuzzy number and $0 \leq \alpha \leq 1$.

$$
\begin{aligned}
&=\frac{1}{4}\left[\int _ { 0 } ^ { 1 } \left\{a_{1}+\alpha\left(a_{2}-\right.\right.\right.\left.\left.a_{1}\right)\right\} d \alpha \\
&+\int_{0}^{1}\left\{a_{3}-\alpha\left(a_{3}-a_{2}\right)\right\} d \alpha \\
&+\int_{0}^{1}\left\{b_{2}-(1-\alpha)\left(a_{2}-b_{1}\right)\right\} d \alpha \\
&\left.+\int_{0}^{1}\left\{b_{2}+(1-\alpha)\left(b_{3}-a_{2}\right)\right\} d \alpha\right], \\
&=\frac{a_{1}+2 a_{2}+a_{3}+b_{1}+2 a_{2}+b_{3}}{8},
\end{aligned}
$$

Therefore,

$$
D^{s}\left(\tilde{A}^{I}, \tilde{0}^{I}\right)=\frac{a_{1}+2 a_{2}+a_{3}+b_{1}+2 a_{2}+b_{3}}{8}, \ldots
$$

\section{SOME THEOREMS/RESULTS RELATED TO $D^{s}\left(\widetilde{A}^{I}, \widetilde{\mathbf{0}}^{I}\right)$ :}

\section{Theorem 1}

$D^{S}\left(k \tilde{A}^{I}, \tilde{0}^{I}\right)=k D^{s}\left(\tilde{A}^{I}, \tilde{0}^{I}\right), k \in \mathbb{R}$, where $D^{s}$ is the sign distance function of TIF number.

Proof:

Case (i) when $k=0$. nothing to prove.

Case (ii) when $k>0$.

Let $\tilde{A}^{I}=\left\{\left(a_{1}, a_{2}, a_{3}\right),\left(b_{1}, a_{2}, b_{3}\right)\right\} \quad$ be a triangular intuitionistic fuzzy number and $k$ be a real number.

$\tilde{A}^{I}=k\left\{\left(a_{1}, a_{2}, a_{3}\right),\left(b_{1}, a_{2}, b_{3}\right)\right\}=$

$\left\{\left(k a_{1}, k a_{2}, k a_{3}\right),\left(k b_{1}, k a_{2}, k b_{3}\right)\right\}$, using property 2

$\therefore k \tilde{A}^{I}=\left\{\left(k a_{1}, k a_{2}, k a_{3}\right),\left(k b_{1}, k b_{2}, k b_{3}\right)\right\}$

Applying $D^{s}$ function, we get

$D^{S}\left(k \tilde{A}^{I}, \tilde{0}^{I}\right)=D^{S}\left(\left\{\left(k a_{1}, k a_{2}, k a_{3}\right),\left(k b_{1}, k a_{2}, k b_{3}\right)\right\}, \tilde{0}^{I}\right)$

$=\frac{k a_{1}+4 k a_{2}+k a_{3}+k b_{1}+k b_{3}}{8}$

$=k \cdot \frac{a_{1}+a_{3}+4 a_{2}+b_{1}+b_{3}}{8}$

$=k D^{S}\left(\tilde{A}^{I}, \tilde{0}^{I}\right)$.

Case (iii) when $k<0$.

Let $\quad \tilde{A}^{I}=\left\{\left(a_{1}, a_{2}, a_{3}\right),\left(b_{1}, a_{2}, b_{3}\right)\right\} \quad$ be a triangular intuitionistic fuzzy number and $k$ be a real number.

$k \tilde{A}^{I}=k\left\{\left(a_{1}, a_{2}, a_{3}\right),\left(b_{1}, a_{2}, b_{3}\right)\right\}=$

$\left\{\left(k a_{3}, k a_{2}, k a_{1}\right),\left(k b_{3}, k a_{2}, k b_{1}\right)\right\}$, using property 2

$\therefore k \tilde{A}^{I}=\left\{\left(k a_{1}, k a_{2}, k a_{3}\right),\left(k b_{3}, k a_{2}, k b_{1}\right)\right\}$

Applying $D^{s}$ function, we get

$$
\begin{aligned}
& D^{s}\left(k \tilde{A}^{I}, \tilde{0}^{I}\right)=D^{s}\left(\left\{\left(k a_{3}, k a_{2}, k a_{1}\right),\left(k b_{3}, k a_{2}, k b_{1}\right)\right\}, \tilde{0}^{I}\right), \\
= & \frac{k a_{3}+k a_{1}+4 k a_{2}+k b_{3}+k b_{1}}{8} \\
= & k \cdot \frac{a_{1}+a_{3}+4 a_{2}+b_{1}+b_{3}}{8} \\
= & k D^{s}\left(\tilde{A}^{I}, \tilde{0}^{I}\right) .
\end{aligned}
$$




\section{Theorem 2}

Let

$$
\tilde{A}^{I}=\left\{\left(a_{1}, a_{2}, a_{3}\right),\left(b_{1}, a_{2}, b_{3}\right)\right\}
$$

and

$\tilde{C}^{I}=\left\{\left(c_{1}, c_{2}, c_{3}\right),\left(d_{1}, c_{2}, d_{3}\right)\right\}$ be two TIF numbers. Then $D^{S}\left(\tilde{A}^{I} \oplus \tilde{C}^{I}, \tilde{0}^{I}\right)=D^{S}\left(\tilde{A}^{I}, \tilde{0}^{I}\right)+D^{S}\left(\tilde{C}^{I}, \tilde{0}^{I}\right)$.

Proof:

$\because \tilde{A}^{I} \oplus \tilde{C}^{I}=\left\{\left(a_{1}+c_{1}, a_{2}+c_{2}, a_{3}+c_{3}\right),\left(b_{1}+d_{1}, a_{2}+\right.\right.$ $\left.\left.c_{2}, b_{3}+d_{3}\right)\right\}$, using Property (P1)

Now applying $D^{s}$ function, we get

$$
\begin{aligned}
& \begin{array}{r}
D^{s}\left(\tilde{A}^{I} \oplus \tilde{C}^{I}, \tilde{0}^{I}\right)=D^{s}\left(\left\{\left(a_{1}+c_{1}, a_{2}+c_{2}, a_{3}+c_{3}\right),\left(b_{1}\right.\right.\right. \\
\left.\left.\left.+d_{1}, a_{2}+c_{2}, b_{3}+d_{3}\right)\right\}, \tilde{0}^{I}\right)
\end{array} \\
= & \frac{a_{1}+c_{1}+a_{3}+c_{3}+4\left(a_{2}+c_{2}\right)+b_{1}+d_{1}+b_{3}+d_{3}}{8} \\
= & \frac{a_{1}+c_{1}+a_{3}+c_{3}+4 a_{2}+4 c_{2}+b_{1}+d_{1}+b_{3}+d_{3}}{8} \\
= & \frac{a_{1}+a_{3}+4 a_{2}+b_{1}+b_{3}}{8}+\frac{c_{1}+c_{3}+4 c_{2}+d_{1}+d_{3}}{8} \\
= & D^{s}\left(\tilde{A}^{I}, \tilde{0}^{I}\right)+D^{s}\left(\tilde{C}^{I}, \tilde{0}^{I}\right) .
\end{aligned}
$$

\section{Theorem 3}

$$
\begin{aligned}
& D^{S}\left(\tilde{A}^{I}, \tilde{0}^{I}\right)=D^{S}\left(\left\{\left(a_{1}, a_{2}, a_{3}\right),\left(b_{1}, a_{2}, b_{3}\right)\right\}, \tilde{0}^{I}\right) \quad \text { is } \quad a_{2} \text { if } \\
& a_{2}-a_{1}=a_{3}-a_{2} \text { and } a_{2}-b_{1}=b_{3}-a_{2} .
\end{aligned}
$$

\section{Proof:}

$D^{S}\left(\tilde{A}^{I}, \tilde{0}^{I}\right)$ of given TIF numbers is

$$
\begin{gathered}
D^{s}\left(\tilde{A}^{I}, \tilde{0}^{I}\right)=\frac{a_{1}+a_{3}+4 a_{2}+b_{1}+b_{3}}{8}, \ldots,(i) \\
a_{2}-a_{1}=a_{3}-a_{2} \Rightarrow a_{2}=\frac{a_{1}+a_{3}}{2},
\end{gathered}
$$

Similarly

$$
a_{2}-b_{1}=b_{3}-a_{2} \Rightarrow a_{2}=\frac{b_{1}+b_{3}}{2},
$$

(i) can be rewritten as

$$
D^{s}\left(\tilde{A}^{I}, \tilde{0}^{I}\right)=\frac{\frac{a_{1}+a_{3}}{2}+2 a_{2}+\frac{b_{1}+b_{3}}{2}}{4}, \ldots,(i i)
$$

Putting the values of $a_{2}$ in (ii), we get

$$
\begin{gathered}
D^{s}\left(\tilde{A}^{I}, \tilde{0}^{I}\right)=\frac{a_{2}+2 a_{2}+a_{2}}{4} \\
=a_{2} .
\end{gathered}
$$

\section{Theorem 4}

Let $\tilde{A}^{I}=\left\{\left(a_{1}, a_{2}, a_{3}\right),\left(b_{1}, a_{2}, b_{3}\right)\right\}$ be triangular intuitionistic fuzzy number. If $a_{1}=b_{1}, b_{3}=a_{3}$. then

$$
D^{S}\left(\tilde{A}^{I}, \tilde{0}^{I}\right)=\frac{a_{1}+2 a_{2}+a_{3}}{4}
$$

which is ranking of triangular fuzzy numbers $\left(a_{1}, a_{2}, a_{3}\right)$.

\section{Proof:}

Let $\tilde{A}^{I}=\left\{\left(a_{1}, a_{2}, a_{3}\right),\left(b_{1}, a_{2}, b_{3}\right)\right\}$ be triangular intuitionistic fuzzy number

Given $a_{1}=b_{1}, b_{3}=a_{3}, \ldots,(i)$

We know that $D^{S}\left(\tilde{A}^{I}, \tilde{0}^{I}\right)$ of triangular intuitionistic fuzzy numbers

$$
D^{s}\left(\tilde{A}^{I}, \tilde{0}^{I}\right)=\frac{a_{1}+a_{3}+4 a_{2}+b_{1}+b_{3}}{8}, \ldots
$$

From $(i)$ and (ii),we get the following result

$$
D^{s}\left(\tilde{A}^{I}, \tilde{0}^{I}\right)=\frac{2 a_{1}+4 a_{2}+2 a_{3}}{8}=\frac{a_{1}+2 a_{2}+a_{3}}{4} .
$$

\section{Theorem 5}

$D^{S}\left(\lambda, \widetilde{0}^{I}\right)=\lambda$, where $\lambda$ is a real number.

\section{Proof:}

We know that

$$
D^{S}\left(\tilde{A}^{I}, \tilde{0}^{I}\right)=\frac{a_{1}+a_{3}+4 a_{2}+b_{1}+b_{3}}{8}, \ldots,(i)
$$

Since $\lambda$ is a real number, $\lambda$ can be written in intuitionistic fuzzy sense as

$\lambda=\{(\lambda, \lambda, \lambda),(\lambda, \lambda, \lambda)\}, \ldots,(i i)$.

From (i), (ii), we have

$D^{s}\left(\lambda, \widetilde{0}^{I}\right)=\lambda$.

Note: In particular if $\lambda=0$, we get

$D^{s}\left(0, \widetilde{0}^{I}\right)=0$.

\section{ALGORITHM FOR PROPOSED METHOD}

The stepwise algorithm of the proposed method for finding the optimal solution of a fully IFLP problem:

$$
\begin{aligned}
\text { Maximize (or Minimize) } Z & =\tilde{c}^{I F} \otimes \tilde{x}^{I F} \\
\text { S.t. } \quad \tilde{a}^{I F} \otimes \tilde{x}^{I F} & =\tilde{b}^{I F} \\
\tilde{x}^{I F} & \geq \tilde{0}^{I F} .
\end{aligned}
$$

In above problem, $\tilde{c}^{I F}, \tilde{a}^{I F}, \tilde{b}^{I F}$, are triangular intuitionistic fuzzy numbers.

Step 1.

If all $\tilde{c}^{I F}, \tilde{a}^{I F}, \tilde{b}^{I F}$ are triangular intuitionistic fuzzy numbers, then we write above fully IFLP problem as: Maximize (or Minimize)

$\tilde{Z}=\sum_{j=1}^{n}\left\{\left(\eta_{j}, \xi_{j}, \zeta_{j}\right),\left(\eta_{j}^{\prime}, \xi_{j}^{\prime}, \zeta_{j}^{\prime}\right)\right\} \otimes\left\{\left(x_{j}, y_{j}, z_{j}\right),\left(p_{j}, y_{j}, r_{j}\right)\right\}$ S.t.

$$
\begin{aligned}
& \text { S.t. } \\
& \begin{aligned}
\sum_{j=1}^{n}\left\{\left(p_{i j}, q_{i j}, r_{i j}\right),\right. & \left.\left(p_{i j}^{\prime}, q_{i j}^{\prime}, r_{i j}^{\prime}\right)\right\} \otimes\left\{\left(x_{j}, y_{j}, z_{j}\right),\left(p_{j}, y_{j}, r_{j}\right)\right\} \\
& =\left\{\left(r_{i}, s_{i}, t_{i}\right),\left(r_{i}^{\prime}, s_{i}, t_{i}^{\prime}\right)\right\}, \\
i & =1,2, \ldots, m, \\
j & =1,2, \ldots, n, \\
x_{j} & \geq 0, \ldots, \text { (3). }
\end{aligned}
\end{aligned}
$$

Step 2.

In this step, we apply properties 1,2 and put $\left\{\left(p_{i j}, q_{i j}, r_{i j}\right),\left(p_{i j}^{\prime}, q_{i j}^{\prime}, r_{i j}^{\prime}\right)\right\} \otimes\left\{\left(x_{j}, y_{j}, z_{j}\right),\left(p_{j}, y_{j}, r_{j}\right)\right\}=$ $\left\{\left(f_{i j}, g_{i j}, h_{i j}\right),\left(m_{i j}, n_{i j}, o_{i j}\right)\right\}$ in fully IFLP problem (3), we get

$$
\tilde{Z}=\sum_{j=1}^{n}\left\{\left(\eta_{j}, \xi_{j}, \zeta_{j}\right),\left(\eta_{j}^{\prime}, \xi_{j}^{\prime}, \zeta_{j}^{\prime}\right)\right\} \otimes\left\{\left(x_{j}, y_{j}, z_{j}\right),\left(p_{j}, y_{j}, r_{j}\right)\right\},
$$


S.t.

$$
\begin{gathered}
\left.\sum_{j=1}^{n}\left(f_{i j}, g_{i j}, h_{i j}\right),\left(m_{i j}, n_{i j}, o_{i j}\right)\right\}=\left\{\left(r_{i}, s_{i}, t_{i}\right),\left(r_{i}^{\prime}, s_{i}, t_{i}^{\prime}\right)\right\}, \\
i=1,2, \ldots, m, \\
j=1,2, \ldots, n, \\
x_{j} \geq 0, \ldots,(4) .
\end{gathered}
$$

Step 3.

Thus the problem (4) is transformed to Maximize (or Minimize) $D^{s}\left(\tilde{Z}, \tilde{0}^{I}\right)$

$$
\begin{aligned}
& =D^{s}\left(\sum_{j=1}^{n}\left\{\left(\eta_{j}, \xi_{j}, \zeta_{j}\right),\left(\eta_{j}^{\prime}, \xi_{j}^{\prime}, \zeta_{j}^{\prime}\right)\right\}\right. \\
& \left.\otimes\left\{\left(x_{j}, y_{j}, z_{j}\right),\left(p_{j}, y_{j}, r_{j}\right)\right\}, \quad \tilde{0}^{I}\right),
\end{aligned}
$$

S.t.

$$
\begin{gathered}
\sum_{j=1}^{n} f_{i j}=r_{i}, \\
\sum_{j=1}^{n} g_{i j}=s_{i}, \\
\sum_{j=1}^{n} h_{i j}=t_{i}, \\
\sum_{j=1}^{n} m_{i j}=r_{i}^{\prime}, \\
\sum_{j=1}^{n} o_{i j}=t_{i}^{\prime}, \\
y_{j}-x_{j} \geq 0, \\
z_{j}-y_{j} \geq 0, \\
y_{j}-p_{j} \geq 0, \\
q_{j}-y_{j} \geq 0, j=1,2, \ldots, n, \ldots,(5) .
\end{gathered}
$$

\section{Step 4}

Solving (5) crisp linear programming in sense IF sets, we get the optimal solution $\left\{\left(x^{0}, y^{0}, z^{0}\right),\left(p^{0}, y^{0}, q^{0}\right)\right\}$ of the considered fully IFLP problem.

\section{Step 5}

Putting these values of decision variables, we get the optimal value of the objective function.

\section{NUMERICAL ILLUSTRATION}

Let fully intuitionistic fuzzy linear programming (FIFLP) problems be given as

$$
\begin{gathered}
\text { Maximize } Z=\{(8,16,24),(0,16,32)\} \otimes \widetilde{X_{1}} \\
\oplus\{(16,24,32),(8,24,40)\} \otimes \widetilde{X_{2}} \\
\text { Such that }\{(1,2,3),(0.5,2,4)\} \otimes \widetilde{X_{1}} \\
\oplus\{(2,3,4),(1,3,5)\} \otimes \widetilde{X_{2}} \\
=\{(3,9,25),(1,9,60)\} \\
\{(1,2,3),(0.5,2,4)\} \otimes \widetilde{X_{1}} \oplus\{(1,2,3),(0.5,2,4)\} \\
\otimes \widetilde{X_{2}}= \\
\{(3,8,24),(1,8,55)\} \\
\widetilde{X_{1}}, \widetilde{X_{2}} \geq \widetilde{0} .
\end{gathered}
$$

Where, $\widetilde{X_{1}}=\left\{\left(x_{1}, y_{1}, z_{1}\right),\left(p_{1}, y_{1}, q_{1}\right)\right\}$,

$\widetilde{X_{2}}=\left\{\left(x_{2}, y_{2}, z_{2}\right),\left(p_{2}, y_{2}, q_{2}\right)\right\}, \quad$ and

$\tilde{0}=\{(0,0,0),(0,0,0)\}$.

We get optimal solution of fully IFLP problem (6) using algorithm described in section (6). Thus the optimal solutions of decision variables are:

$\tilde{X}_{1}^{0}=\{(3,7,7),(2,3,8.75)\}, \tilde{X}_{2}^{0}=\{(0,1,1),(0,1,5)\} . \quad$ And optimal value of the intuitionistic fuzzy objective function is $\tilde{Z}_{1}^{0}=\{(24,72,200),(0,72,480)\}$.

It is clear that the fully acceptable value of objective function is 72 . Membership degree is increasing from 24 to 72 and then decreasing from 72 to 200 and values 0,480 are totally unacceptable. Also, it can be observed in the sketch of membership and non-membership functions of objective are given below in fig. 3 .

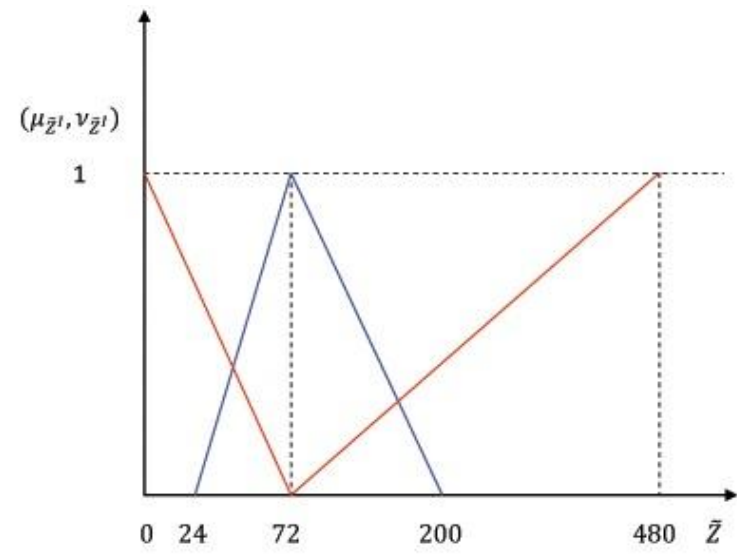

Figure 3: Membership and non-membership function of intuitionistic fuzzy objective.

\section{CONCLUSION}

Thus in this paper, we have considered some properties of positive triangular intuitionistic fuzzy numbers. Further, we have considered intuitionistic fuzzy origin and computed distance of an intuitionistic fuzzy numbers from intuitionistic fuzzy origin. Using the concept of sign distance, we have proposed new method to find the optimal solution of fully intuitionistic fuzzy linear programming problem. In this problem, we have considered all the parameters such as cost coefficients, coefficient matrix and resource vectors as TIFNs. Proposed method is easy to handle the fully intuitionistic fuzzy linear programming problem and thus may be considered a suitable method for solving FIFLP problems.

\section{ACKNOWLEDGMENTS}

Authors are thankful to University Grants Commission, Government of India, New Delhi for providing the financial support for the research work.

\section{REFERENCES}

[1] Atanassov T. Intuitionistic fuzzy sets, Fuzzy Sets and Systems, Vol.20 pp.87-96, 1986. 
[2] Angelov, P. P., Optimization in an intuitionistic fuzzy environment, Fuzzy Sets and Systems, vol. 86, pp. 299306, 1997.

[3] Bellman, R. E., Zadeh, L.A. Decision making in a fuzzy environment, Management Science, Vol.17, pp. B141B164, 1970.

[4] Dubey, Dipti, Mehra, Aparna, Linear programming with Triangular Intuitionistic Fuzzy Number", EUSFLATLFA 2011, Advances in Intelligent Systems Research, Atlantis Press, Vol.1 , No.1, pp. 563-569, 2011.

[5] Bharati, S. K. and Singh, S.R., Solving Multi-Objective Linear Programming Problems Using Intuitionistic Fuzzy Environment Optimization Method: a Comparative Study, International Journal of Modeling and Optimization, DOI: 10.7763/IJMO.2014.V4.339.

[6] Li, D. F., A ratio ranking method of triangular intuitionistic fuzzy numbers and its application to MADM problems, J. Computer and Mathematics with Applications 60, (2010), 1557-1570.

[7] Mitchell, H. B., Ranking intuitionistic fuzzy numbers, International J. of Uncertainty, Fuzziness and Knowledge-Based Systems, Vol. 12, No.3, (2004), 377386.

[8] Zadeh, L.A., Fuzzy Sets, Information and control, Vol. 8 ,pp.338-353, 1965.

[9] Cheng, Ching-Hsue, A new approach for ranking fuzzy numbers by distance method, Fuzzy Sets and Systems 95 (1998) 307-317.

[10] Yao, J. S, Wu K., Ranking fuzzy numbers based on decomposition principle and sign distance, Fuzzy Sets and systems 116 (2000) 275-288.

[11] Tran Lien, Duckstein, Comparison of fuzzy number using a fuzzy measure, Fuzzy Sets and systems 116 (2002) 331-341.

[12] Chu, Ta-Chung, Tsao, Chung-Tsen, Ranking fuzzy numbers with an area between the centroid point and original point, Computers and mathematics with Applications 43 (2002) 111-117.

[13] Grzegorzewski, P., Distance between intuitionistic fuzzy sets and interval-valued fuzzy sets based on the hausdorff on the Hausdorff metric, Fuzzy Sets and Systems 148 (2004) 319-328.

[14] Abbasbandy, S., Asady, B., Ranking of fuzzy numbers by sign distance, Information Sciences 176 (2006) 24052416.
[15] Shureshjani, R. S., Darehmiraki M., A new parametric method for ranking fuzzy numbers, Indagationes Mathematicae 24 (2013) 518-529.

[16] Szmidt, E., Kacprzyk J., Distance between intuitionistic fuzzy sets, Fuzzy Sets and Systems 114 (2000) 505-518.

[17] Wang, W., Xin, X., Distance measure between intuitionistic fuzzy sets, Pattern Recognition Letters 26 (2005) 2063-2069.

[18] Nayagam, G. V, Venketshwary, G., Shivaraman, G., Ranking of intuitionistic fuzzy numbers, 2008 IEEE International Conference on Fuzzy Systems (FUZZ 2008).

[19] Li1, D. F., Nan, J. X., Zhang, M. J., A Ranking method of triangular intuitionistic fuzzy numbers and application to decision making, International Journal of computational Intelligence Systems Nol.2, No.5, (2010), 522-530.

[20] Nayagam1G. V., Sivaraman, V. G., Modified ranking of intuitionistic fuzzy numbers, NIFS 17 (2011) 1, 5-22.

[21] Wei, C. P., Tang, X., Possibility degree method for ranking intuitionistic fuzzy numbers, 2010 IEEE/WIC/ACM.

[22] Nehi, H. M., A new ranking method for intuitionistic fuzzy numbers, International Journal of Fuzzy Systems, Vol. 12, No.1, 2010

[23] De, P. K., Das, D., A study on ranking of trapezoidal intuitionistic fuzzy numbers, 6 (2014), 437-444.

[24] Zhang, H., Yu, L., New distance measures between intuitionistic fuzzy sets and interval-valued fuzzy sets, Information Sciences 245 (2013) 181-196.

[25] Guha D., Chakrborty D., A theoretical development of distance measure for intuitionistic fuzzy numbers, International Journal of Mathematics and Mathematica Sciences, 2010

[26] Esmailzadeh, M., Esmailzadeh, M., New distance between triangular intuitionistic fuzzy numbers, Advances in Computational Mathematics and Its Applications vol. 2, no.3, (2013).

[27] Yao1, J.S., Lin, F.T., Fuzzy critical path method based on signed distance ranking of fuzzy numbers, IEEE Transaction on Systems, Man and Cybernatics-Part- A: Systems and Humans Vol.30, No.1, 2000.

[28] Zimmermann, H. J., Fuzzy programming and linear programming with several objective functions, Fuzzy Sets and Systems, 1(1978) 45-55. 